$\S=-1$

\title{
Interference Avoidance Using Dynamic Frequency Reuse For LTE-A System
}

\author{
Asif Reza ${ }^{1}$, Md. Rafiqul Islam ${ }^{2}$, Khaizuran Bin Abdullah ${ }^{3}$, Farah Raisa ${ }^{4}$ \\ Department of Electrical \& Computer Engineering, International Islamic University Malaysia \\ 53100 Kuala Lumpur, Malaysia \\ *CorrespondingauthorE-mail: asifrezaaust@gmail.com;rafiq@iium.edu.my,khaizuran@iium.edu.my,farah.raisa.luma@gmail.com
}

\begin{abstract}
To meet the increasing demand for spectrum in communication system, Long Term Evolution (LTE) system has been proposed. It allowed users to use a new and much wider spectrum comparing to the other previous technologies. To utilize the full capacity of the LTE system Frequency Reuse (FR) has been adapted. It is a promising aspect of transmission of high rate data stream with better system capacity and fading immunity in the modern Long-Term Evolution-Advanced (LTE-A) system. Fractional Frequency Reuse (FFR) is the commonly used frequency reuse technique to increase the system capacity. But the problem is, with introduction of FR, it also increases the Inter Cell Interference (ICI) of the system. The out of cell interference coming from the neighbouring e-NodeBs (eNB) as a result of using the same frequency band and they act as an interference source. This ICI decreases the system capacity, resulting in hampering of communication, browsing and in worst case no connection at all. This condition highly affects the users under a cell specially those who are located in the cell edge areas. To take care of this issue an approach called Dynamic Frequency Reuse scheme has been proposed in this paper to mitigate the interference thus increasing the system capacity. This scheme uses continuous assessment of resource allocation and choosing the best sub-band for the user with least interference. There is a sequential order to be maintained for the users with using off minimum Base Station (BS) transmit power. The cell edge area users are the primary concern in terms of increasing their service quality. MATLAB simulation software has been used to justify the theory that the proposed scheme is capable increase the capacity of the cell edge areas as well as the cell centre area user. The results achieved from the simulation also indicates that the proposed model can enhance the system capacity comparing to the existing FFR scheme.
\end{abstract}

\section{Introduction}

The demand for system capacity is increasing everyday due to the increasing in internet data traffic. The number of mobile users is also increasing in a fast rate. There is a statistic from European Mobile Observatory (EMO) that is indicating that 92 percent of mobile is rising every year since the year of 2006 [1]. There is also a prediction that, within the next decades the mobile traffic will increase by 1000 times comparing to the present day [2]. So, to overcome the demand for capacity there is a need for modification of the currently deployed Long Term Evolution (LTE) technology which is commonly known as " $4 \mathrm{G}$ " (Fourth Generation).

The idea of Long Term Evolution Advanced (LTE-A) was proposed to meet the requirement of the users and also the suppliers. The recent researches are indicating that LTE-A will be able to provide high data rate of $1 \mathrm{Gbps}$. To further improve the condition of LTE-A Frequency Reuse concept has been adapted in LTE-A system and transforming the network from Homogeneous to Heterogeneous Network (HetNet) is a promising aspect that can significantly improve the system capacity.

Like the LTE system, LTE-A has also adapted the Orthogonal Frequency Division Multiple Access (OFDMA) technique. OFDMA is the newest technology of accessing in mobile communication system. However, implementation of HetNet and FR concept has some demerits too. It can increase the Inter Cell Interference (ICI) of the system as the neighbouring cells use the same frequency band. So, the neighbouring eNB may act as an interference source to each other. This is a limiting factor that many researchers are interested in mitigating.

Traditional Fractional Frequency Reuse is an effective solution of this problem. Fractional frequency reuse is a combination of reuse- 1 and reuse-N model. In this scheme, each cell is divided into two regions. One is cell centre area and other one is cell edge area [3]. Frequency reuse factor of 1 is used for the cell centre area.

In reuse-1 scheme, users situated in each cell can access the whole bandwidth but the problem is, it has to deal with the inter cell interference from the neighbouring cell. On the other hand, the cell edge areas use a higher frequency reuse scheme. In this case the bandwidth gets reduced but the inter cell interference is less. This is a trade-off. Based on these experiments the fractional frequency reuse method is applied. Here, the available spectrum is divided into two sub-bands. One of them is used in cell centre area and other one is used in cell edge area permanently. As they are operating on disjoint spectrum, the capacity of the cell edge area is improved comparing to the universal reuse. This is an important scheme to avoid ICI. In this paper, for the sake of simplicity, a two-cell model has been considered.

However, the disadvantages of this scheme are, a portion of the available spectrum is a portion of the available spectrum is re- 
maining unused. Also, it cannot handle with changing user traf In order to overcome these problems, the method called Dynaimu Frequency Reuse (DFR) is proposed. This method is applied under different parametric condition and realistic scenarios.

The paper is organised as follows, in section 2 some related works are discussed. Section 3 is about the description of the proposed model. In section 4 results and discussions are presented and finally conclusion and future work is added in the section 5 .

\section{Related Work}

Application of fractional frequency reuse in the LTE-A system has been a highly-discussed topic in communication society. Adaptive partial frequency reuse has been proposed in [4] can improve the system capacity. However different transmit power and positions have been assumed arbitrarily. In another paper [5], contention based FFR is proposed but the algorithm is applicable for certain conditions only. In [6], congestion management in intellectual transportation system is proposed. Soft Frequency Reuse (SFR) has been proposed in [7-9]. However, most of the paper of them do not consider the effect of inter cell interference. Dynamic allocation of frequency is considered in [10]. However, none of these considered multiple base stations with each serving users that are located randomly.

\section{Overview of Proposed Model}

The proposal of initial dynamic frequency reuse includes two steps of initialization. The first is implementation of an empirical, circulated algorithm for ideal resource allocation in LTE-A system. When there is no chance of communication with other BS, each BS can dynamically assign resource for each of its users. But assigning resources allocation to improve the capacity leads to [11] Non-Deterministic Polynomial Time Hard (NP-Hard) problem. A problem is considered NP-hard, if an algorithm for solving it can be translated into one for solving any NP problem. So, NP-Hard means it is as hard as any NP problem although it can be even harder. Due to this problem, computational complexity arises. In order to avoid this, a proposal has been made in this paper called Dynamic Frequency Reuse scheme. The total power allocated is minimized with a constant data rate for each of the user by resources allocated by BS. The idea is to group the subcarriers into sub-bands. In telecommunication system, the subcarriers are signal carrier that is carried on top of another carrier. As a result, two signals can be carried at the same time. At the receiving end, the main carrier and the subcarrier signals are demodulated separately. On the other hand, the sub-band is the sub-division of a frequency band and sub-band coding, typically done by Fast Frequency Transformation (FFT) breaks the signal into different frequency bands. In other word, it can be represented by the set of subcarriers. For a user to be placed in an exact sub-band means that his virtual channel will include only frequencies that are part of that sub-band.

The following assumptions were made, the OFDMA in LTE-A system consists of inter-cell interference but there is no intercarrier interference. There is a constant data rate, $R$ for users. They only require this rate, $R$ for maintain the services. Channel quality information is fed back for each sub-band and user.

If the channel quality information is denoted as, $i$ and the calculated subcarrier is $m_{i j}$ and the amount of required power is $p_{i j}$, then the user would need the CDR, $R$ if user $i$ is placed in subband $j$. Let's consider a binary random variable $Z_{i j}$ which indicates, if user $i$ is allotted to sub-band $j$. Then, the following constrained integer programming problem will be solved in each BS,

$$
\sum p_{i j} z_{i j} \leq p
$$

$$
\sum_{i} m_{i j} Z_{i j} \leq c \forall_{j}
$$

In this Paper, $C$ is sub-band capacity and $p$ is total power constraint. This equation can be further relaxed by allowing $Z_{i j}$ to be real in the interval $[0,1]$ and $\sum_{j} z_{i j}=1, \forall_{i}, z_{i j} \geq 0 \forall_{i, j}$ in $Z_{i j}$ 's tends to be 0 or 1 .

Here, A two cell scenarios is considered. When cell edge users placed in cell 1 are allocated to sub-band which is 1 , the cell 2 users will face high interference on sub-band 1 and it will automatically be pushed towards sub-band 2. Cell centre users are not as much affected by ICI as the cell edge users. So, it will choose the sub-band with best condition. In this case, sequential order is maintained for the users. The user's COST is maintained by the base station. COST is a function of the price for required power on sub-band. In other word, it can be mentioned that COST is a function of power. Cost minimized means the low power sub-band but that can maintain the desired service of the user without any interruption. State variable of the base station monitors the available power and the sub-band in the base station. This data is taken and updated after that next user is taken in account. When the allocation period ends, the state variables are reduced to represent the work orders.

\section{Results \& Discussion}

For analyzing the results, following assumptions are made;

Table 1 : Parameters considered

\begin{tabular}{|l|l|}
\hline Parameters & Value \\
\hline Path loss model & $\begin{array}{r}\text { COST 231-Walfisch Ikagami model } \\
42.64+26 \log 10(d)+20 \log (f)\end{array}$ \\
\hline Inter site distance & $2.5 \mathrm{Km}$ \\
\hline Band Width & $20 \mathrm{MHz}$ \\
\hline Constant data rate & $12 \mathrm{Kbps}$ \\
\hline No. of subcarriers & 48 \\
\hline $\begin{array}{l}\text { Subcarriers allocated } \\
\text { for each user }\end{array}$ & 1 \\
\hline Fading condition & Rayleigh fading \\
\hline Thermal Noise Power & $P=K T B$, Here $K=$ Botlzman Constant \\
\hline
\end{tabular}

Considering the above-mentioned parameters, simulations has been done using Matlab. In the previous section, the capacity of reuse- 1 and reuse- $\mathrm{N}$ has been discussed. The combination of these to reuse factors give the capacity of the FFR scheme. The capacity of the FFR scheme is shown in Figure 1(a). Here, the capacity of FFR scheme can be seen at different power levels. When the power level is increasing the capacity also increasing with the distance. In this paper, the cell edge users are given more priorities as they are mainly affected by the ICI scenario.

In the simulation for our proposed model, a limit has been considered for the users to be assigned in the sub-bands and power consumption. The simulation will stop when there is lack of subcarriers or power. User's placement and fading has been considered multiple times. Then the data are analyzed and stored for the use of simulation and take care of randomness. New users are added from the different locations. Figure 2 shows the system capacity based on the number of users supported using DFR comparing to the FFR. From the simulation results, it is clear that DFR outperforms the FFR.

Furthermore, if the base station transmit power is kept constant we can evaluate the performance of DFR comparing to FFR by Figure 2 with data analysis is presented below. Table 2 of comparison of data analysis of indicating the enhancement of capacity in DFR. 


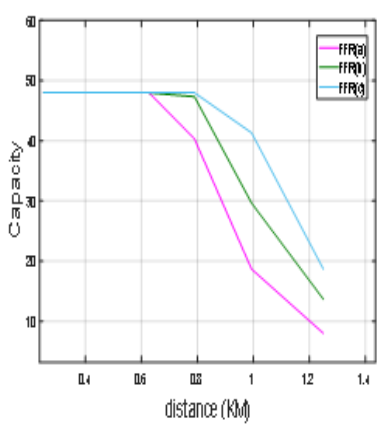

(a)

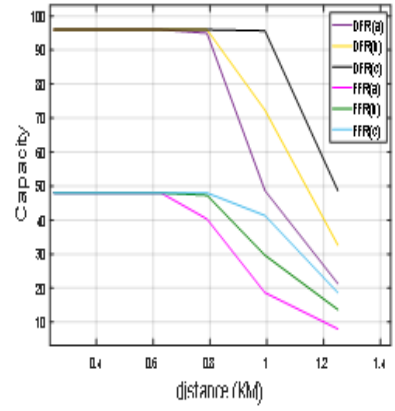

(b)
Figure 1: Performance of (a) FFR \& (B) DFR Comparing to FFR

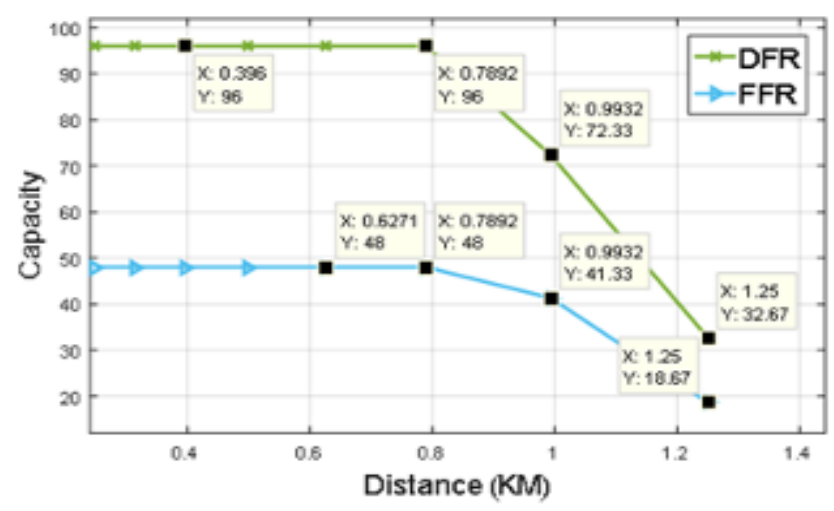

Figure 2: Data Analysis of DFR vs FFR

Table 2: Comparison of DFR vs FFR

\begin{tabular}{|l|l|l|l|}
\hline Distance (KM) & DFRCapacity & FFR Capacity & Difference \\
\hline 0.4 & 96 & 48 & 48 \\
\hline 1 & 72 & 41.33 & 30.6 \\
\hline 1.25 & 32 & 18.6 & 13.4 \\
\hline
\end{tabular}

\section{Conclusion}

The FFR is a promising aspect in order to reduce the ICI of the system but it also arises some problems. To reduce the effect of ICI, DFR scheme is proposed. The proposed scheme compares the available sub-bands and then chooses the best sub-band for the users. Simulations results also indicates that this scheme outperforms the traditional FFR scheme. At the cell edge area and also the cell center area, the capacity is almost double comparing to FFR. In the simulation, the environment is considered with the realistic parameters. Further studies regarding this matter may lead to efficient radio resource management.

\section{Acknowledgement}

The authors are grateful to Research Management Center (RMC), International Islamic University Malaysia (IIUM) to support this research through initiative grant scheme RIGS 16-065-0229

\section{References}

[1] Wang, Cheng-Xiang, Haider, Fourat, Gao, Xiqi, You, Xiao-Hu, Yang, Yang, Yuan, Dongfeng, Aggoune, Hadi M., Haas, Harald, Fletcher, Simon, Hepsaydir, Erol. "Cellular architecture and key technologies for 5G wireless communication networks." IEEE Communications Magazine 52.2 (2014): 122-130.
[2] Qian Clara Li, Huaning Niu, Apostolos Tolis Papathanassiou, Geng Wu. "5G network capacity: key elements and technologies." IEEE Vehicular Technology Magazine 9.1 (2014): 71-78. Sun, Chen, Jian Shu, Wei Zheng, Zhiyong Yang, and Xiaojun Wang. "Adaptive partial frequency reuse in LTE-Advanced relay networks." In 2015 IEEE 34th International Performance Computing and Communications Conference (IPCCC), pp. 1-8. IEEE, 2015.

[3] Chen Sun, Jian Shu, Wei Zheng, Zhiyong Yang and Xiaojun Wang, "Adaptive partial frequency reuse in LTE-Advanced relay networks," 2015 IEEE 34th International Performance Computing and Communications Conference (IPCCC), Nanjing, 2015, pp. 1-8.

[4] Zhan, Hao-Yue, Bin-Jie Hu, Zong-Heng Wei, Wen-Ji Liu, Bing Li, and Xing Liu. "Contention-based fractional frequency reuse scheme in LTE/LTE-A network." In Information Technology, Networking, Electronic and Automation Control Conference, IEEE, pp. 663-666. IEEE, 2016.

[5] Ali, Nora A., Mohamed A. El-Dakroury, Magdi El-Soudani, Hany M. ElSayed, Ramez M. Daoud, and Hassanein H. Amer. "New hybrid frequency reuse method for packet loss minimization in LTE network." Journal of advanced research 6, no. 6 2015: 949-955.

[6] Yu, Yiwei, Eryk Dutkiewicz, Xiaojing Huang, Markus Mueck, and Gengfa Fang. "Performance analysis of soft frequency reuse for inter-cell interference coordination in LTE networks." In Communications and Information Technologies (ISCIT), 2010 International Symposium on, pp. 504-509. IEEE, 2010.

[7] Qian, Manli, Wibowo Hardjawana, Yonghui Li, Branka Vucetic, Xuezhi Yang, and Jinglin Shi. "Adaptive soft frequency reuse scheme for wireless cellular networks." IEEE Transactions on Vehicular Technology 64, no. 1 2015: 118-131.

[8] Huang, Jinjing, Jiandong Li, Linjing Zhao, and Sheng Huang. "CoSFR: coordinated soft frequency reuse for OFDMA-based multi-cell networks with non-uniform user distribution." Wireless Networks: 1-14 2016.

[9] Rahman, Md Tashikur, Md Didarul Alam, and Mostafa Zaman Chowdhury. "Interference mitigation and capacity enhancement based on dynamic frequency reuse for femtocell networks." In 2015 IEEE International Conference on Telecommunications and Photonics (ICTP), pp. 1-5. IEEE, 2015.

[10] Chung, Sungmoon. "Dynamic Frequency Reuse Scheme Based on Traffic Load Ratio for Heterogeneous Cellular Networks." The Journal of Korean Institute of Communications and Information Sciences 40, no. 12 2015: 2539-2548.

[11] Ye, Qiaoyang, Beiyu Rong, Yudong Chen, Mazin Al-Shalash, Constantine Caramanis, and Jeffrey G. Andrews. "User association for load balancing in heterogeneous cellular networks." IEEE Transactions on Wireless Communications 12, no. 6 2013.: 2706-2716. 